\title{
The interaction between metabolic rate, habitat choice, and resource use in a polymorphic freshwater species
}

\author{
Matilda Andersson ${ }^{1}$, Kristin Scharnweber ${ }^{1}$, and Peter Eklöv ${ }^{1}$ \\ ${ }^{1}$ Uppsala University
}

October 27, 2021

\begin{abstract}
1. Resource polymorphism is common across taxa and can result in alternate ecotypes with specific morphologies, feeding modes, and behaviours that increase performance in a specific habitat. This can result in high intraspecific variation in the expression of specific traits and the extent to which these traits are correlated within a single population. Although metabolic rate influences resource aquisition and the overall pace of life of individuals it is not clear how metabolic rate interact with the larger suite of traits to ultimately determine individual fitness. 2. We examined the relationship between metabolic rates and the major differences (habitat use, morphology, and resource use) between littoral and pelagic ecotypes of European perch (Perca fluviatilis) from a single lake in Central Sweden. 3. Standard metabolic rate (SMR) was significantly higher in pelagic perch but did not correlate with resource use or morphology. Maximum metabolic rate (MMR) was not correlated with any of our explanatory variables or with SMR. Aerobic scope (AS) showed the same pattern as SMR, differing across habitats, but contrary to expectations, was lower in pelagic perch. 4. This study helps to establish a framework for future experiments further exploring the drivers of intraspecific differences in metabolism. In addition, since metabolic rates scale with temperature and determine predator energy requirements, our observed differences in SMR across habitats will help determine ecotype-specific vulnerabilities to climate change and differences in top-down predation pressure across habitats.
\end{abstract}

\section{Hosted file}

Andersson2021_manuscript.docx available at https://authorea.com/users/390981/articles/ 543206-the-interaction-between-metabolic-rate-habitat-choice-and-resource-use-in-apolymorphic-freshwater-species 